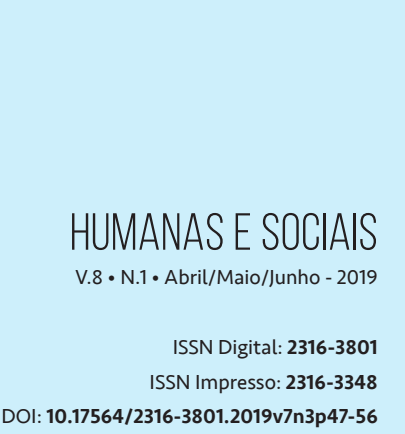

\section{PREVALÊNCIA DE ANSIEDADE EM ESTUDANTES DE MEDICINA DE ALAGOAS}

PREVALENCE OF ANXIETY IN MEDICAL STUDENTS FROM ALAGOAS

\section{LA PREVALENCIA DE LA ANSIEDAD EN LOS ESTUDIANTES DE MEDICINA DE ALAGOAS}

Jorge Alves dos Santos Júnio ${ }^{r 1}$ Diego Victor Belo Lima²

Solange Buarque Tenório ${ }^{3}$

Andressa Pereira Lopes ${ }^{4}$ André Fernando de Oliveira Fermoseli ${ }^{5}$

\section{RESUMO}

Indivíduos na fase adulta frequentemente deparam-se com diferentes situações ambientais que acabam funcionando como propulsores para aparição de sintomas ansiogênicos, especialmente em ambiente universitário, onde existem demandas externas e internas acerca do futuro profissional. Esta pesquisa teve como objetivo verificar a prevalência de ansiedade em estudantes de Medicina de uma instituição privada de Alagoas. Foi aplicado um questionário sociodemográfico, e o Inventário de Ansiedade de Beck (BAI - Beck Anxietylnventory) em 41 estudantes universitários do curso de Medicina. Os resultados mostraram uma incidência baixa entre os estudantes com sintomas ansiosos de moderado a grave, contrariando a maioria das pesquisas. Espera-se que os resultados possam fornecer subsídios para uma melhor compreensão de como traços de personalidade podem influenciar no aparecimento de sintomas ansiosos.

\section{PALAVRAS-CHAVE}

Ansiedade. Estudantes Universitários. Medicina. 


\section{ABSTRACT}

Individuals in adulthood often encounter different environmental situations that end up acting as propellants for the appearance of anxiogenic symptoms, especially in a university environment, where there are external and internal demands on the professional future. This study aimed to verify the prevalence of anxiety in medical students of a private institution in Alagoas. A sociodemographic questionnaire and the Beck Anxiety Inventory (BAI) were applied to 41 undergraduate medical students. The results showed a low incidence among students with moderate to severe anxiety symptoms, contrary to most research. It is hoped that the results may provide insights for a better understanding of how personality traits can influence the onset of anxious symptoms.

\section{KEYWORDS}

Anxiety. College Students. Medicine.

\section{RESUMEN}

Individuos adultos a menudo enfrentan a diferentes situaciones ambientales que terminan actuando como propulsores para la aparición de síntomas ansiogénicos, especialmente en el entorno universitario, donde hay demandas externas y sobre el futuro profesional interna. Esta investigación pretende comprobar la prevalencia de ansiedad en estudiantes de medicina de una institución privada de Alagoas. Se aplicó un cuestionario demográfico y el Inventario de Ansiedad de Beck (BAI - Beck Anxiety Inventory) en 41 estudiantes de grado en medicina. Los resultados mostraron una baja incidencia entre los estudiantes con síntomas de ansiedad de moderados a severos, contrariamente a la mayoría de las investigaciones. Se espera que los resultados puedan proporcionar subsidios para una mejor comprensión de cómo los rasgos de personalidad pueden influir en el surgimiento de los síntomas hacia adelante.

\section{PALABRAS CLAVE}

Ansiedad. Estudiantes universitarios. Medicina. 


\section{INTRODUÇ̧̃̃o}

Esta pesquisa é um recorte de um trabalho de conclusão de curso intitulado Relação entre traços de personalidade e ansiedade em estudantes universitários. Um dos maiores problemas de saúde da atualidade é a ansiedade. De acordo com Barros e “outros autores" (2003) a ansiedade é expressa organicamente por sensação de mal-estar psíquico, que pode resultar em perturbações somáticas como as cardio-respiratórias, opressão e a sensação de ter "nós no estômago e na garganta", além de um estado geral de hipercontração muscular.

Para Margis e "outros autores" (2003), os indivíduos na fase adulta com frequência deparam-se com diferentes situações ambientais que acabam funcionando como propulsores para aparição desses sintomas, como o relacionamento de casal, relações interpessoais, necessidade de manutenção do emprego e aposentadoria etc. Estas situações geram ansiedade e estresse e estão ligadas ao modo de vida do homem contemporâneo.

A ansiedade é um quadro normal e está presente na vida das pessoas e pode surgir nas mais diversas situações. No entanto, torna-se patológica quando esta sintomatologia foge de controle, está presente em grande parte do tempo e traz prejuízos para a vida do sujeito, seja no trabalho, na vida acadêmica, na família ou impedindo este sujeito de ter uma vida social plena.

Os transtornos de ansiedade têm seu começo geralmente na adolescência e início da fase adulta, tendo uma prevalência maior entre os mais jovens. As mulheres têm três vezes mais possibilidades de desenvolver transtornos de ansiedade (SOUZA, 2010). Dados de uma pesquisa multicêntrica realizada na Grande São Paulo com uma amostra de 5.037 adultos mostrou que 19,9\% apresentam transtornos ansiosos, seguido de $11 \%$ que possuem algum transtorno de humor (ANDRADE et al., 2012).

Uma população em que tem sido encontrado alta incidência de ansiedade é a de estudantes universitários. Bandeira e "outros autores" (2005) em uma pesquisa realizada com 135 universitários e os resultados indicaram uma maior presença de sintomas ansiosos nos estudantes dos cursos das ciências humanas em comparação com os alunos dos cursos de ciências exatas. Ferreira e "outros autores" (2009) pesquisaram a incidência de sintomas ansiosos em estudantes universitários em diferentes áreas do conhecimento. 0 estudo constatou que estudantes da área biomédica eram mais ansiosos em comparação com os estudantes de outras áreas. Segundo estes autores as demandas acadêmicas, como uma elevada carga horária, o cumprimento de atividades como monitoria e aulas extras durante os finais de semana seriam fatores propulsores ao aparecimento de sintomas de ansiedade.

Outros estudos também afirmam que os estudantes da área médica são acometidos com frequência com sintomas ansiosos. Baldassin, Martins e Andrade (2006) realizaram uma pesquisa com 481 estudantes de medicina na Grande São Paulo e constataram que 79,9\% dos estudantes possuíam traços de ansiedade média e pelo menos $20,1 \%$ possuíam ansiedade alta. Ahmed e "outros autores" (2009) em um estudo realizado com estudantes de medicina em Dubai, utilizando o Inventário Beck de Ansiedade (BAI) encontraram a prevalência de sintomas ansiosos em 28,7\% dos 165 estudantes de medicina investigados. 
A ansiedade pode gerar uma série de consequências negativas, como transtornos de ansiedade, abuso de álcool e outras drogas e o surgimento de outras comorbidades. Sendo assim estudar a prevalência de ansiedade nos estudantes universitários pode trazer dados importantes para que no futuro ajudem na elaboração de estratégias que visem prevenir esses sintomas e comportamentos que podem interferir no desempenho acadêmico. Desta forma, este estudo teve como objetivo verificar a prevalência de ansiedade em estudantes universitários do curso de Medicina.

\section{METODOLOGIA}

\subsection{PARTICIPANTES}

A pesquisa foi realizada em uma Instituição de Ensino Superior privada, na cidade de Maceió, em 41 estudantes do curso de medicina nos anos iniciais.

\subsection{INSTRUMENTOS}

\subsubsection{INVENTÁRIO DE BECK DE ANSIEDADE- BAI - [CUNHA, 2001]}

É um instrumento validado de autopreenchimento, que contém 21 questões de múltipla escolha, perguntas de autoavaliação sobre a percepção de ansiedade dos indivíduos participantes ao longo da semana anterior.

\subsection{QUESTIONÁRIO DE LEVANTAMENTO DE DADOS DEMOGRÁFICOS DOS PARTICIPANTES}

Questionário sociodemográfico composto por 8 (oito) itens, que foram elaborados pelos pesquisadores. As perguntas estão relacionadas ao perfil dos estudantes em relação a: sexo, idade, curso, período, profissão e estado civil.

\subsection{PROCEDIMENTO}

O projeto foi submetido à Plataforma Brasil e após a aprovação do Comitê de Ética em Pesquisa (parecer 963.762 de 25/02/2015), os pesquisadores agendaram dia e hora com a coordenação do curso, a fim de apresentar os objetivos da pesquisa e agendar um dia para apresentar o projeto para os alunos em sala de aula.

Após as instruções gerais, foi realizada a aplicação dos instrumentos, aconteceu de maneira coletiva na sala de aula. A etapa seguinte foi a entrega dos instrumentos na seguinte ordem: 0 questionário de levantamento de dados demográficos e o Inventário de ansiedade de Beck (BAI) para cada participante. 


\section{ANÁLISE DOS DADOS}

Por se tratar de um estudo quantitativo, a análise dos dados foi realizada com a utilização do software estatístico Statistical Package for Social Science(SPSS) 21 para Windows. Foi criado um banco de dados para efetuar as análises descritivas das variáveis sociodemográficas da amostra e da ansiedade, em seguida, foi realizada a correlação de Pearson para avaliar a correlação entre ansiedade e os dados sociodemográficos.

\section{RESULTADOS E DISCUSSÃO}

A amostra foi composta por 41 estudantes, desses, a maioria era do sexo feminino $(n=24)$, com média de idade $(M=19,53 ; D P=2,05)$. Esses dados estão representados na Tabela 1.

Por meio dos dados obtidos pelo Inventário de Beck de Ansiedade (BAI), observa-se que os estudantes da área de saúde apresentaram os seguintes níveis de ansiedade: mínimo (36\%), leve (37\%), moderado (16\%) e grave (11\%).

Tabela 1 - Frequência quanto ao grau de ansiedade nos estudantes de Medicina

\begin{tabular}{c|c}
\hline Mínimo & 14 \\
\hline Leve & 16 \\
\hline Moderado & 6 \\
\hline Grave & 5 \\
\hline Total & 41 \\
\hline
\end{tabular}

Fonte: Dados de pesquisa

Esse resultado sugere que os estudantes parecem não interpretar a situação atual como ansiogênica, contrariando as pesquisas que revelam que os estudantes de medicina apresentam um grau de ansiedade elevado. Esse fato pode ser explicado em razão da coleta de dados ter ocorrido em um período com ausência de provas e trabalhos. Cruz e "outros autores" (2010) relatam que há uma relação entre a ansiedade dos estudantes universitários e as demandas acadêmicas. Em concordância com isto, Martinez e "outros autores" (2000 apud OLIVEIRA; DUARTE, 2004), refere que as demandas acadêmicas de apresentação de trabalho, seminário e falar para um grupo podem aumentar a ansiedade. No seu estudo, observou-se que 90,69\% dos estudantes universitários apresentavam medo ao falar em público ou lidar com superiores.

Ao analisar a correlação idade e ansiedade (TABELA 2), observa-se que a correlação foi positiva, porém não significativa estatisticamente ( $r p=0,023 ; p>0,05)$. 
Tabela 2 - Correlação entre idade e ansiedade nos estudantes do curso de medicina

\begin{tabular}{c|c}
\hline Curso & Coeficiente \\
\hline Medicina & 0,023 \\
\hline
\end{tabular}

Fonte: Dados de pesquisa.

Devemos considerar que a variação de idade dos participantes foi entre 17 e 27 anos, o que não sugere presença da terceira idade neste estudo, fase na qual, segundo De Oliveira e "outros autores" (2006), são mais frequentes os sintomas de ansiedade, devido aos sentimentos característicos do envelhecer.

\section{CONSIDERAÇÕES FINAIS}

Este estudo buscou investigar a prevalência de ansiedade em estudantes de medicina. Diante disso, conclui-se que a presença de sintomas ansiosos de médio a grave deu-se num número pequeno, inferior ao esperado. 0 resultado da nossa pesquisa difere do amplamente difundido na literatura, que descreve que os estudantes universitários da área médica apresentam níveis elevados de ansiedade, contudo, isso pode ser explicado pelo fato dos alunos não estarem em um período de intensa demanda acadêmica, fator este responsável pelo aparecimento de sintomas ansiogênicos.

Esta pesquisa possui limitações que merecem ser elencadas. A primeira delas é que por se tratar de um estudo transversal, os estudantes não foram acompanhados por um determinado período, o que pode ter influenciado nos resultados desta pesquisa, tornando necessários estudos que visem acompanhar os estudantes da área da saúde por um determinado período. A segunda limitação é a composição da amostra, tendo em vista que foi formada por alunos de períodos iniciais, portanto é pertinente a realização de novas investigações com amostras compostas por alunos no início, no meio e no final do curso.

Diante dos resultados obtidos e das limitações apresentadas neste estudo, destaca-se a necessidade de pesquisas que tenham como foco estudantes universitários com o intuito fornecer subsídios para elaboração de medidas que visem prevenir o surgimento de ansiedade e comportamentos capazes interferir no desempenho acadêmico.

\section{REFERÊNCIAS}

AHMED, I. Cognitive emotions: depression and anxiety in medical students and staff. Journal of critical care, v. 24, n. 3, p. e1-e7, 2009. Disponível em: http://www.jccjournal.org/article/S08839441(09)00134-8/abstract. Acesso em: 28 out. 2016. 
ANDRADE, L. H. et al. Mental Disorders in Megacities: Findings from the São Paulo Megacity Mental Health Survey, Brazil. Plos One, v. 7, n. 2, 2012. Disponível em: http://journals.plos.org/plosone/ article?id=10.1371/journal.pone.0031879. Acesso em: 2 dez. 2016.

BALDASSIN, S.; MARTINS, L. C.; ANDRADE, A. G. de. Traços de ansiedade entre estudantes de Medicina. Arquivos Médicos do ABC, v. 31, n.1, p. 27-31, 2006. Disponível em: https://www. portalnepas.org.br/amabc/article/view/232/228. Acesso em: 29 fev. 2016.

BANDEIRA, M. et al. Comportamento assertivo e sua relação com ansiedade, lócus de controle e auto-estima em estudantes universitários. Estudos de Psicologia, Campinas, v. 22, n. 2, p. 111-121, 2005.

BARROS, A. L. B. L. de. Situações geradoras de ansiedade e estratégias para seu controle entre enfermeiras: estudo preliminar. Revista Latino-americana de Enfermagem, v. 11, n. 5, p. 585-92, 2003. Disponível em: http://www.scielo.br/pdf/rlae/v11n5/v11n5a04. Acesso em: 28 dez. 2016.

CRUZ, C. M. V. M. et al. Ansiedade nos estudantes do ensino superior. Revista Millenium, n. 38, p. 223-242. 2010. Disponível em: http://www.ipv.pt/millenium/Millenium38/15.pdf. Acesso em: 1 dez. 2016.

CUNHA, J. A. Manual da versão em português das Escalas Beck. São Paulo: Casa do Psicólogo, 2001.

DE OLIVEIRA, K. L. et al. Relação entre ansiedade, depressão e desesperança entre grupos de idosos. Psicologia em Estudo, v. 11, n. 2, p. 351-359, 2006. Disponível em: http://www.scielo.br/ pdf/pe/v11n2/v11n2a13.pdf. Acesso em: 3 dez. 2016.

FERREIRA, C. L. et al. Universidade, contexto ansiogênico? Avaliação de traço e estado de ansiedade em estudantes do ciclo básico. Ciência e saúde coletiva, v.14, n. 3, Rio de Janeiro, maio/jun. 2009. Disponível em: http://www.scielosp.org/scielo.php?script=sci_arttext\&pid =S1413-81232009000300033. Acesso em: 28 out. 2016.

MARGIS, Regina et al. Relação entre estressores, estresse e ansiedade. Revista de psiquiatria do Rio Grande do Sul, Porto Alegre, v. 25, supl. 1, abr. 2003. Disponível em: http://www.scielo.br/scielo. php?script=sci_arttext\&pid=S0101-81082003000400008\&lng=en\&nrm=iso. Acesso em: 29 nov. 2016.

OLIVEIRA, M. A. de; DUARTE, A. M. M. Controle de respostas de ansiedade em universitários em situações de exposições orais. Revista Brasileira de Terapia Comportamental e Cognitiva, v. 6, 
n. 2, p. 183-199, 2004. Disponível em: http:// pospsicopatologia.com.br/angela/controle_de_ respostas_de_ansiedade.pdf. Acesso em: $1 \mathrm{dez}$. 2016.

SOUZA, L. Prevalência de sintomas depressivos, ansiosos e estresse em acadêmicos de medicina. 2010. 233f. Tese (Doutorado em Ciências) Faculdade de Medicina, Universidade de São Paulo, São Paulo, 2010. Disponível em: http:// www.incor.usp.br/sites/incor2013/docs/egressosteses/2011/Jan_2011_Luciano_Tese.pdf. Acesso em: 24 set. 2016.
Recebido em: 28 de Janeiro de 2019

Avaliado em: 2 de Maio de 2019

Aceito em: 2 de Maio de 2019

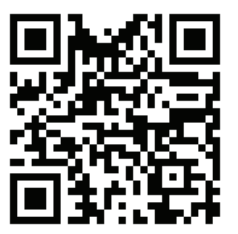

A autenticidade desse artigo pode ser conferida no site https://periodicos. set.edu.br

1 Acadêmico do curso de Mestrado do Programa de pós-graduação em Psicologia da Universidade Federal de Alagoas - UFAL. E-mail: jorge.junior@ip.ufal.br

2 Psicólogo pelo Centro Universitário Tiradentes - UNIT/AL. E-mail: diegovictors@hotmail.com

3 Psicóloga pelo Centro Universitário Tiradentes - UNIT/AL. E-mail: solangebuarque@uol.com.br

4 Doutora em Psicologia Clínica pela Unicap; Profesora Titular do curso de Psicologia do Centro Universitário Tiradentes - UNIT/AL. E-mail: andressa_lopes@hotmail.com

5 Doutor em Ciências pela USP; Prof. Titular do curso de Psicologia do Centro Universitário Tiradentes - UNIT/AL. E-mail: afermoseli@hotmail.com

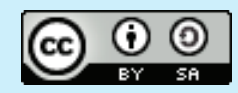

Este artigo é licenciado na modalidade acesso abertosob a Atribuição-Compartilhalgual CC BY-SA

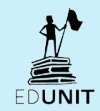

